\title{
Mixed optical phonon modes in semiconductor nanocrystals synthesized in porous $\mathrm{Al}_{2} \mathrm{O}_{3}$ matrix
}

\author{
A. I. Belogorokhov ${ }^{1}$, L. I. Belogorokhova ${ }^{2}$, R. P. Miranda ${ }^{3}$, M. I. Vasilevskiy ${ }^{* 3}$, and S. A. \\ Gavrilov $^{4}$ \\ ${ }^{1}$ GIREDMET State Scientific Center, B. Tolmachevskii per., 119017 Moscow, Russia \\ ${ }^{2}$ Physics Department, M. V. Lomonosov Moscow State University, 119899 Moscow, Russia \\ ${ }^{3}$ Centro de Física, Universidade do Minho, 4710-057 Braga, Portugal \\ ${ }^{4}$ Moscow Institute for Electronic Engineering (Technical University), 103498 Moscow, Russia
}

Received 17 July 2004, revised 21 July 2004, accepted 6 September 2004

Published online 17 November 2004

PACS 63.22.+m, 78.30.Fs

This work is devoted to experimental and theoretical studies of optical phonons confined in CdS nanocrystals grown inside a porous alumina matrix. The complete sets of phonon modes, confined in a spherical quantum dot and in a cylindrical quantum wire, are considered in the framework of a continuum model, which allows the mixing of all the expected types of vibrations. It is shown that the calculated phonon-related response in the far-infrared spectral region agrees qualitatively with the measured spectra of typical samples, providing an explanation for a rather unusual structure of the reflection and transmission spectra.

() 2004 WILEY-VCH Verlag GmbH \& Co. KGaA, Weinheim

\section{Introduction}

Optical phonons confined in semiconductor nanostructures have attracted much attention in the last few years [1-3] since they are crucial in determining the electronic and optical properties of the low-dimensional systems. As known, because of the mechanical and electrostatic confinement in these structures, the energies of the vibrational eigenmodes are shifted to values different from the bulk material and new phonon modes, localized at the interfaces, can emerge [1]. Both the confined bulk-like and interface phonon modes have already been observed experimentally [4].

There are several experimental techniques which have been successfully applied in the fabrication of semiconductor nanocrystallites (NC's) [1]. Studies of porous materials produced by electrochemical etching have proven that these systems look promising candidates to act as matrices for the NC's [5]. Owing to the typical topology characteristic of these materials, with voids of different shapes and sizes, the NC's can also have different shapes ranging from nearly spherical quantum dots (QD's) to elongated rods which can be considered as quantum wires (QW's).

In this work we consider optical phonons confined in spherical QD's and long cylindrical QW's in the framework of a continuum model, which has proven to be successful for QD's [6]. We calculate phononrelated response in the far-infrared (FIR) spectral region and compare it to experimental results obtained on CdS NC's grown inside a porous alumina (por- $\mathrm{Al}_{2} \mathrm{O}_{3}$ ) matrix.

\footnotetext{
* Corresponding author: e-mail: mikhail@ fisica.uminho.pt
} 


\section{Experimental details}

The samples of CdS NC's were synthesized inside a por- $\mathrm{Al}_{2} \mathrm{O}_{3}$ matrix produced by etching, using a joint consideration of a potential-pH diagram with cyclic voltammetry data to optimize the conditions of $\mathrm{CdS}$ cathodic deposition (see Ref. [7] for a detailed discussion of this method).

The electrolyte solutions were prepared by dissolving $\mathrm{Na}_{2} \mathrm{~S}_{2} \mathrm{O}_{3}$ and $\mathrm{CdSO}_{4}$ in deionized water. The $\mathrm{pH}$ value of the solution was adjusted by using $\mathrm{H}_{2} \mathrm{SO}_{4}$ or $25 \% \mathrm{NH}_{4} \mathrm{OH}$. An aqueous solution of 0.01 $\mathrm{M} \mathrm{CdSO}_{4}+0.01 \mathrm{M} \mathrm{Na}_{2} \mathrm{~S}_{2} \mathrm{O}_{3}$ was used as the base mixture for $\mathrm{CdS}$ deposition, which was performed in a three-electrode system. Two $1 \times 1 \mathrm{~cm}^{2} \mathrm{Pt}$ plates were used as the working and counter electrode, and saturated calomel as the reference electrode, separately. The potential sweep rate in the cyclic voltammetry was kept at $10 \mathrm{mV} / \mathrm{s}$. Both the control potential deposition and cyclic voltammetry studies were carried out at room temperature and in unstirred solutions. Porous anodic alumina films of $1 \mu \mathrm{m}$ thickness were used as templates for CdS QD's and QW's electrodeposition. The anodic film was formed in $20 \% \mathrm{H}_{2} \mathrm{SO}_{4}$ aqueous solution under a $2 \mathrm{~mA} / \mathrm{cm}^{2}$ current density during $2000 \mathrm{~s}$.

Turning to the spectroscopic results, photoluminescence spectra of the samples (not shown here) were considerably blue-shifted with respect to the bulk material, which is a manifestation of the quantum confinement effect. This energy shift allowed us to estimate the typical size of the CdS NC's, which was found to be in the range of 2.5-9.7 nm for different samples, in good agreement with the results obtained from Atomic Force Microscopy measurements. Raman spectra of the samples (also not shown here) typically contained an asymmetrically broadened peak near the longitudinal-optical (LO) phonon frequency of the bulk material $\left(\omega_{L O}\right)$, as it is common of semiconductor NC's. On the contrary, the FIR reflection and transmission spectra revealed a rather rich structure which differs from both bulk and QD's. Figure 1 shows the FIR reflection and optical density spectra for a typical sample. It is clear from the figure the presence of absorption bands at frequencies right below $\omega_{L O}$ and close to the transverse-optical (TO) phonon frequency of the bulk material $\left(\omega_{T O}\right)$. One can notice some wavy patterns in both spectra which probably are just interference fringes. Still, we can speculate the presence of FIR activity near the Fröhlich frequency [8] (see Fig. 1b), which is given by $\omega_{F}=\left[\left(\varepsilon_{s} \omega_{L O}^{2}+2 \varepsilon_{h} \omega_{T O}^{2}\right) /\left(\varepsilon_{s}+2 \varepsilon_{h}\right)\right]^{1 / 2}$, where $\varepsilon_{s}$ and $\varepsilon_{h}$ denote the high-frequency dielectric constants of the semiconductor material and the surrounding medium, respectively.

While absorption bands near $\omega_{F}$ are typical of QD's and FIR activity at frequencies close to $\omega_{T O}$ can be explained by the occurrence of QD clustering [6], the feature in the vicinity of $\omega_{L O}$ seems puzzling at first sight.
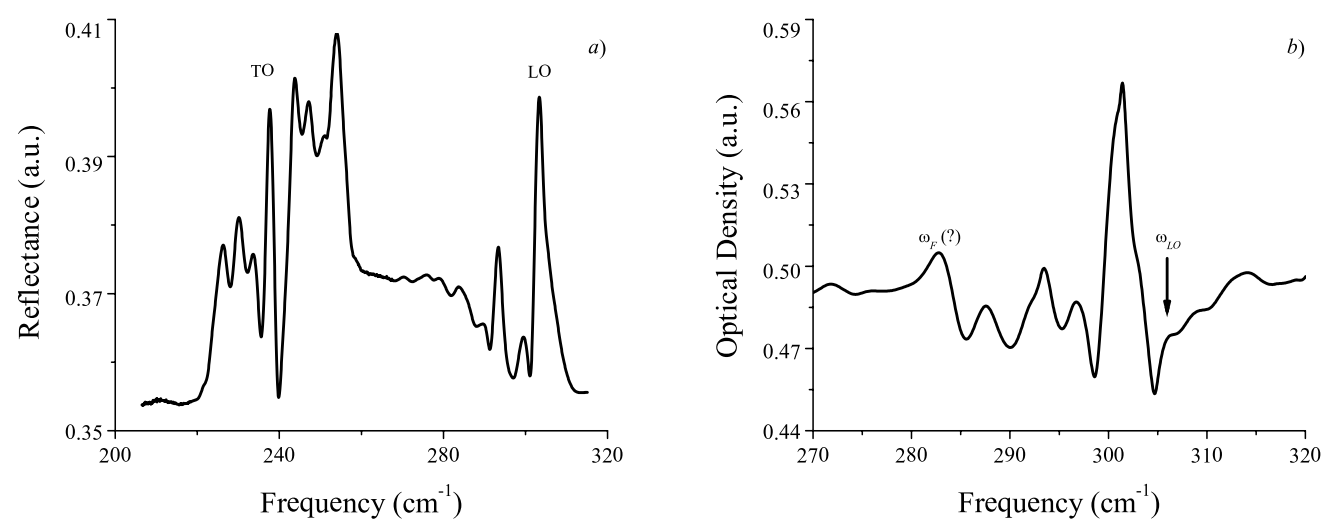

Fig. 1 FIR reflection (a) and optical density (b) spectra obtained for a typical sample of CdS NC's embedded in por- $\mathrm{Al}_{2} \mathrm{O}_{3}$. 


\section{Confined dipolar modes in semiconductor QD's and QW's}

In order to find the vibrational eigenmodes of semiconductor heterostructures it is necessary to solve the phenomenological equation of motion [6,9] coupled to the Poisson equation. Solving these equations for a sphere with radius $R_{Q D}$ and for a cylinder with radius $R_{Q W}$ and length $L \gg R_{Q W}$, allows one to determine the complete set of phonon modes in QD's and QW's, which includes confined longitudinal and transverse (with two different polarizations) vibrations and also interface modes. Mechanical and electrostatic boundary conditions mix these three types of vibrations in the FIR-active modes, which are distributed throughout the whole reststrahlen band of the semiconductor material. Details of such a calculation for QD's can be found in Ref. [6]. For QW's, the details will be published elsewhere.

Given the vibrational eigenmodes, it is possible to determine the polarizability of a single NC. The expressions for the polarizability of a single QD can be found in Ref. [6]. For a single QW, the components of the polarizability (which is a tensor in this case) can be written as

$$
\alpha_{\|}^{Q W}=\frac{\varepsilon_{s}-\varepsilon_{h}}{\varepsilon_{h}} \frac{V_{Q W}}{4 \pi}+\frac{\varepsilon_{s} \rho\left(\omega_{L O}^{2}-\omega_{T O}^{2}\right)}{4 \pi \varepsilon_{h}} \sum_{\substack{\text { modes with } \\ m=0 \text { and } q \sim 0}} \frac{\left|A_{n}\right|^{2}}{\omega_{n}^{2}-\omega^{2}-\mathrm{i} \Gamma \omega_{n}}
$$

and

$$
\alpha_{\perp}^{Q W}=\frac{\varepsilon_{s}-\varepsilon_{h}}{\varepsilon_{s}+\varepsilon_{h}} \frac{V_{Q W}}{2 \pi}+\frac{\varepsilon_{s} \varepsilon_{h} \rho\left(\omega_{L O}^{2}-\omega_{T O}^{2}\right)}{\pi\left(\varepsilon_{s}+\varepsilon_{h}\right)^{2}} \sum_{\substack{\text { modes with } \\ m= \pm 1 \text { and } q \sim 0}} \frac{\left|B_{n}\right|^{2}}{\omega_{n}^{2}-\omega^{2}-\mathrm{i} \Gamma \omega_{n}}
$$

where $V_{Q W}$ and $\rho$ denote, respectively, the volume and the reduced mass density of the QW, $\Gamma$ is the phonon damping parameter, and $q, n, m$ are appropriate quantum numbers for QW's. $\left|A_{n}\right|^{2}$ and $\left|B_{n}\right|^{2}$ are oscillator strengths given by expressions similar to those for QD's (see Ref. [6]).

For the larger radii $\left(R_{Q D, Q W}>1.5-2 \mathrm{~nm}\right)$, the combined effect of many confined modes is similar to the macroscopic model prediction. For QD's, there should be a single peak in $\operatorname{Im} \alpha^{Q D}$ situated at $\omega_{F}$. For QW's, Im $\alpha_{\perp}^{Q W}$ should exhibit a single peak at $\omega_{F}^{\prime}=\left[\left(\varepsilon_{s} \omega_{L O}^{2}+\varepsilon_{h} \omega_{T O}^{2}\right) /\left(\varepsilon_{s}+\varepsilon_{h}\right)\right]^{1 / 2}$, which is the two-dimensional analogous of the Fröhlich frequency in spherical QD's. Notice that, as we consider infinitely long QW's, the quantum confinement effect only manifests itself in the radial direction.

The dielectric properties of an ensemble containing both QD's and QW's can be described by means of an effective dielectric function, $\varepsilon^{*}$, which is related to the FIR absorption of the ensemble [6]. If the filling fractions of the QD's, $f_{Q D}$, and of the QW's, $f_{Q W}$, are small, then the Maxwell-Garnett approximation provides an adequate description of this average dielectric function. Assuming Gaussian distributions of QD's and QW's radii with mean values and standard deviations given by $\bar{R}_{Q D}, \sigma_{Q D}$ and $\bar{R}_{Q W}, \sigma_{Q W}$, respectively, the effective dielectric function can be calculated using the expression:

$$
\frac{\varepsilon^{*}-\varepsilon_{h}}{\varepsilon^{*}+2 \varepsilon_{h}}=\frac{4 \pi}{3}\left\{f_{Q W} \frac{\int F_{Q W}(R) \bar{\alpha}^{Q W} \mathrm{~d} R}{\int F_{Q W}(R) V_{Q W} \mathrm{~d} R}+f_{Q D} \frac{\int F_{Q D}(R) \alpha^{Q D} \mathrm{~d} R}{\int F_{Q D}(R) V_{Q D} \mathrm{~d} R}\right\},
$$

where $F_{i}(R)=\exp \left[-\left(R-\bar{R}_{i}\right)^{2} /\left(2 \sigma_{i}^{2}\right)\right] /\left(\sqrt{2 \pi} \sigma_{i}\right)(i=Q D, Q W)$ and $\bar{\alpha}^{Q W}=\left(\alpha_{\|}^{Q W}+2 \alpha_{\perp}^{Q W}\right) / 3$. Figure 2 shows the imaginary part of $\varepsilon^{*}$ calculated for composites containing an $f_{Q D}+f_{Q W}=0.05$ volume fraction of CdS NC's of two different mean radii. It is clear from the figure that the quantum confinement effect becomes less important as the NC mean radius increases.

\section{Discussion and conclusion}

The results obtained in the previous section show that the FIR absorption spectra of composites containing both QD's and QW's of very small radii should exhibit a well-defined multi-mode structure with resonances 

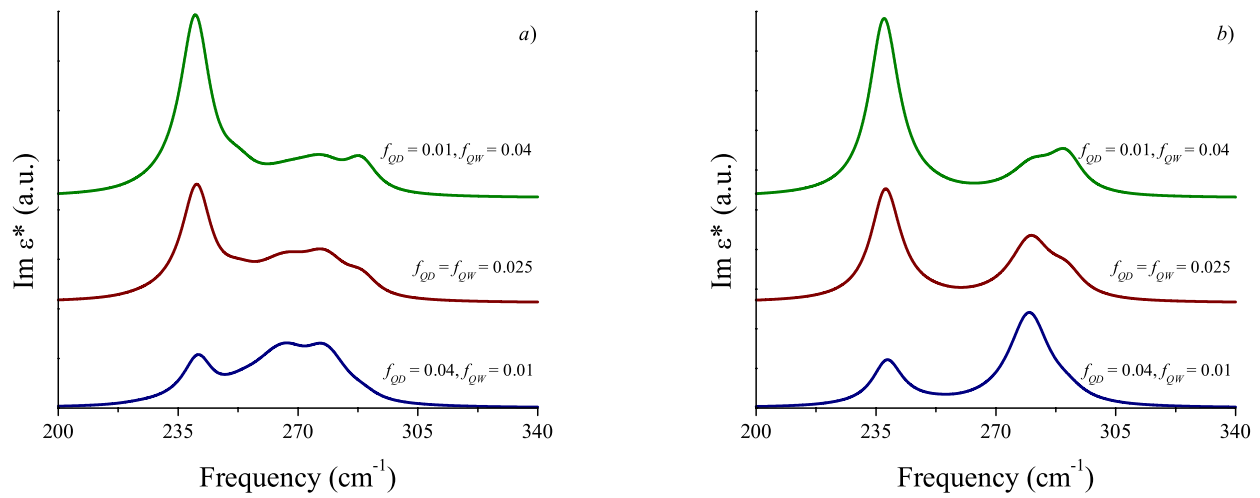

Fig. 2 Imaginary part of the effective dielectric function of composites containing CdS QD's and QW's with mean radii $\bar{R}_{Q D}=\bar{R}_{Q W}$ of $1 \mathrm{~nm}$ (a) and $3 \mathrm{~nm}$ (b) and a relative standard deviation of $10 \%$, embedded in a matrix with $\varepsilon_{h}=1.5$. The filling fractions are indicated on the figure.

associated with the few dipolar modes allowed in such small spheres and cylinders. When the NC radius becomes sufficiently large, the quantum size effect disappears and the absorption spectrum recovers its classical form.

When the NC's are embedded in a porous material, like in our experiment, the voids are only partly filled with QD's and QW's. Therefore, the dielectric constant of the host medium is just an effective parameter which accounts for the presence of the matrix. We used the value $\varepsilon_{h}=1.5$, but it could be even lower. In any case, this effective dielectric constant is always much lower than that of the semiconductor material and, so, $\omega_{F}^{\prime}$ lies very close to $\omega_{L O}$. We can say that our calculated results agree qualitatively with the measured spectra, in the sense that if the volume fraction of the CdS QW's is higher than that of the QD's (which probably is the case), the vibrational eigenmodes of the QW's with frequencies close to $\omega_{F}^{\prime}$ clearly produce a peak right below $\omega_{L O}$. Also, the absorption bands observed near $\omega_{T O}$ can be explained by the resonance at this frequency due to the vibrational eigenmodes of the CdS QW's, together with the occurrence of QD clustering.

Acknowledgements This work was supported by the Portuguese Foundation for Science and Technology (FCT) through project POCTI/FIS/10128/98 and by the Russian Foundation for Basic Research under grant 03-02-16938.

\section{References}

[1] D. Bimberg, M. Grundmann, and N. N. Ledentsov, Quantum Dot Heterostructures (John Wiley \& Sons, Chichester, 1999).

[2] T. D. Krauss, F. W. Wise, and D. B. Tanner, Phys. Rev. Lett. 76, 1376 (1996).

[3] M. I. Vasilevskiy et al., phys. stat. sol. (b) 224, 599 (2001).

[4] B. Jusserand and M. Cardona, in: Nonlinear Optics of Random Media, edited by M. Cardona and G. Güntherodt (Springer-Verlag, Hedelberg, 1989).

[5] A. I. Belogorokhov and L. I. Belogorokhova, Phys. Sol. State 43, 1693 (2001).

[6] M. I. Vasilevskiy, Phys. Rev. B 66, 195326 (2002).

[7] S. A. Gavrilov, S. Y. Tikhonova, E. A. Zhukov, A. I. Belogorokhov, and L. I. Belogorokhova, in: Proc. 25th Int. Conf. on the Physics of Semiconductors, edited by N. Miura and T. Ando (2000), pp. 1745-1746.

[8] R. Ruppin and R. Englman, Rep. Prog. Phys. 33, 149 (1970).

[9] C. Trallero-Giner, F. Garcia-Moliner, V. R. Velasco, and M. Cardona, Phys. Rev. B 45, 11944 (1992). 